\title{
Uso do brinquedo terapêutico no cuidado ao acesso venoso em pediatria: um relato de experiência
}

Use of therapeutic toy in the care of pediatric patients with venous access: an experience report

Uso de juguete terapéutico en el cuidado de pacientes pediátricos con acceso venoso: un informe de experiencia

Lorena Gomes de Araújo ${ }^{1 *}$, Janaína Mendes de Melo¹, Elizabeth Pinheiro Araújoㄹ, Paula Regina Barbosa de Almeida ${ }^{1}$, Andressa Tavares Parente ${ }^{1}$, Edficher Margotti ${ }^{1}$, Angeline do Nascimento Parente $^{1}$, Thamyles da Silva Dias ${ }^{1}$, Sheila Barbosa Paranhos ${ }^{1}$, Joyce Regina Pereira ${ }^{1}$.

\section{RESUMO}

Objetivo: Relatar a experiência de acadêmicas de enfermagem na utilização do Brinquedo Terapêutico como estratégia de educação aos pais e/ou responsáveis e crianças hospitalizadas quanto aos cuidados com os acessos venosos em pediatria. Relato de experiência: Trata-se de um estudo descritivo, do tipo relato de experiência, realizado a partir da vivência de acadêmicas de enfermagem do sexto semestre de uma instituição de ensino superior pública, na realização do cuidado pediátrico através do Brinquedo Terapêutico, durante a prática da atividade Curricular de Enfermagem Pediátrica, no mês de setembro de 2019, em uma enfermaria pediátrica de um hospital de referência na capital do estado do Pará. A elaboração do plano de ação, confecção de folder e bonecos sincronicamente atendeu a demanda do tema, através da atividade de educação em saúde usando o Brinquedo Terapêutico. Considerações finais: É notório os benefícios do emprego do brinquedo terapêutico como recurso para educar em pediatria. A ferramenta favoreceu a compreensão quanto à importância do procedimento e contribui para esclarecimento dos cuidados com o acesso venoso, o que torna a internação menos traumática, e ainda contempla o direito da criança hospitalizada. A adesão a essa intervenção positiva continua sendo um desafio no processo de hospitalização pediátrica.

Palavras-chave: Enfermagem pediátrica, Jogos e brinquedos, Cuidados de enfermagem, Educação em saúde.

\begin{abstract}
Objective: To report the experience of nursing students in the use of the Therapeutic Toy as an education strategy for parents and/or guardians and their hospitalized children during the care of pediatrics patients with venous accesses. Experience report: This is a descriptive study, based in a experience report, carried out from the experience of nursing students from the sixth semester of a public higher education institution, during pediatric care using the Therapeutic Toy, in the practice of the Pediatric Nursing Curriculum activity, in September 2019, in a pediatric ward of a reference hospital in the capital city of the state of Pará, Brazil. The elaboration of the action plan, making of folders and puppets synchronously met the demand of the theme, through the health education activity using the Therapeutic Toy. Final considerations: The benefits of using therapeutic toys as a resource for educating in pediatrics are notorious. The tool favored the understanding of the importance of the procedure and contributes to clarifying the care of pediatric patients with venous accesses, which makes hospitalization less traumatic, and also contemplates the rights of hospitalized children. Adherence to this positive intervention remains a challenge in the pediatric hospitalization process.
\end{abstract}

Keywords: Pediatric nursing, Play and playthings, Nursing care, Health education.

\section{RESUMÉN}

Objetivo: Relatar la experiencia de estudiantes de enfermería en el uso del Juguete Terapéutico como estrategia educativa para padres y/o tutores y niños hospitalizados sobre el cuidado del acceso venoso en pediatría. Informe de experiencia: Se trata de un estudio descriptivo, del tipo informe de experiencia, realizado a partir de la experiencia de estudiantes de enfermería del sexto semestre de una institución pública

1 Universidade Federal do Pará (UFPA), Belém - PA. *E-mail: lorena.lga1999@gmail.com 
de educación superior, en la realización de la atención pediátrica a través del Juguete Terapéutico, durante la práctica del Actividad del Currículo de Enfermería Pediátrica, en septiembre de 2019, en una sala de pediatría de un hospital de referencia en la capital del estado de Pará, Brazil. La elaboración del plan de acción, confección de folletos y muñecos atendió sincrónicamente a la demanda de la temática, a través de la actividad de educación para la salud utilizando el Juguete Terapéutico. Consideraciones finales: Son notorios los beneficios de utilizar juguetes terapéuticos como recurso para la educación en pediatría. La herramienta favoreció la comprensión de la importancia del procedimiento y contribuye a esclarecer la atención con acceso venoso, lo que hace que la hospitalización sea menos traumática, y también contempla el derecho de los niños hospitalizados. La adherencia a esta intervención positiva sigue siendo un desafío en el proceso de hospitalización pediátrica.

Palabras clave: Enfermería pediátrica, Juego e implementos de juego, Cuidados de enfermería, Educación en salud.

\section{INTRODUÇÃO}

O acesso venoso é o procedimento de introdução de um dispositivo de invasivo, denominado cateter, comumente usados em hospitalizações com finalidades de monitorização hemodinâmica, nutrição parenteral, manter uma via de infusão de solução e medicamentos. Contudo, o local da inserção se torna uma porta de entrada para infecções, por esse motivo é necessária a atenção redobrada aos cuidados com o local da punção (SILVA PR, et al., 2017).

No processo de hospitalização pediátrica, considerado um processo atípico, doloroso e traumático, frente à criança, que necessita de cuidados específicos relacionados às diversas patologias, são realizados diversos procedimentos invasivos, dentre eles o acesso venoso. Para uma criança é um momento repleto de sentimentos, como medos, incertezas, estresse e angústia; os quais nem sempre é capaz de compreender. Esse contexto soma-se ao fato de estar inserida em um ambiente estranho, distante do seu convívio social e familiar. Desse modo, é essencial humanizar a hospitalização para amenizar a experiência, de forma deixála menos traumática, e tornar a criança ativa no seu processo de saúde-doença. Para isso, uma estratégia usada é a incorporação de atividades lúdicas - descontração, diálogos infantis, brincadeiras, entre outros (ROCKEMBACH J, et al., 2017; SANTOS BRF, et al., 2020).

O brincar faz parte do ser criança e está relacionado ao desenvolvimento motor, emocional, mental e social, e a importância é reafirmada no Estatuto da Criança e do Adolescente, disposta no artigo 16, item IV. Nessa perspectiva, surge o Brinquedo Terapêutico (BT), que é uma tecnologia de cuidado por meio da brincadeira estruturada, capaz de auxiliar na diminuição da ansiedade decorrente da situação, na facilitação da atenção prestada, na aceitação do tratamento, no estabelecimento da comunicação, no envolvimento na terapêutica e nos procedimentos, na manutenção dos direitos da criança e na (re)significação da doença (CALEFFI CCF, et al., 2016; VEIGA MAB, et al., 2016).

O BT pode ser classificado de três formas, sendo o Brinquedo Terapêutico Dramático que objetiva a manifestações de sentimento, descarga emocional, desejos e experiências da criança; Brinquedo Terapêutico Capacitador de Funções Fisiológicas que permite aprender sobre a readaptação da função fisiológica para sua nova condição de vida; e o Brinquedo Terapêutico Instrucional usado para orientar e facilitar no preparo da criança para o procedimento, através da participação no manuseio dos materiais e realização do mesmo antes pela criança (CALEFFI CCF, et al., 2016; PENNAFORTL VPS, et al., 2018).

São explícitos os diversos benefícios proporcionados pela aplicação do BT à criança, dessa forma, são fundamentais que este instrumento não se restrinja apenas a um único local, mas que seja utilizado sempre que a criança apresente alguma dificuldade de enfrentamento, alguma situação ameaçadora ou necessite de preparação para a realização de procedimentos (JULIANI RML e SOUZA AS, 2019).

Em março de 2005 foi proposta a Lei 11.104, que torna obrigatória a criação de brinquedoteca em hospitais que possuam atendimento pediátrico em regime de internação. Essa medida demonstra que as atividades lúdicas contribuem para o processo de melhoria da saúde das crianças, auxiliando no enfrentamento desse momento da vida e a adaptação infantil ao ambiente hospitalar (BRASIL, 2005). 
Nesse enfoque, o Conselho Federal de Enfermagem (COFEN) aprova a Resolução no 546/2017 que torna atribuição do enfermeiro atuante na área pediátrica a utilização do Brinquedo Terapêutico na assistência à criança e à família hospitalizadas, seguindo todas as etapas do processo de enfermagem. Reafirmando a necessidade da brinquedoterapia em pacientes infantis, como um agente que contribui no processo saúde e doença, visto que compreender o contexto cultural que a família e as crianças estão inseridas deve ser estratégia importante para implementar um plano de cuidados eficaz (COFEN, 2017).

Diante do exposto, definiu-se como objetivo do presente estudo relatar a experiência de acadêmicas de enfermagem na utilização do Brinquedo Terapêutico como estratégia de ensino aos pais e/ou responsáveis e crianças hospitalizadas quanto aos cuidados com os acessos venosos em pediatria.

\section{RELATO DE EXPERIÊNCIA}

Trata-se de um estudo descritivo, do tipo relato de experiência, realizado a partir da vivência de acadêmicas de enfermagem do sexto semestre de uma Instituição de Ensino Superior Pública no estado do Pará, com enfoque no cuidado pediátrico fundamentado no uso da ferramenta do BT durante a prática da atividade Curricular de Enfermagem Pediátrica, no mês de setembro de 2019, em uma enfermaria pediátrica de um hospital de referência materno infantil na capital do estado do Pará, Belém-PA. A enfermaria conta com 28 leitos, dos quais 24 encontravam-se ocupados e participaram da ação, que integrou as crianças e os pais/responsáveis. O tema da ação foi definido a partir da problemática percebida pelos acadêmicos durante a vivência no campo e discutida com a equipe assistencial da enfermaria pediátrica. Os cuidados com acessos venosos (periféricos e centrais) na pediatria predominaram como indicação.

A primeira etapa foi destinada para o planejamento da ação. As acadêmicas se reuniram com a equipe do projeto de extensão, cujo tema é "a utilização do brinquedo terapêutico como instrumento da assistência no cuidado cirúrgico pediátrico", para discutir sobre os aspectos legais e científicos BT e seus benefícios no processo de hospitalização da criança.

Na segunda foi estruturado um plano de ação com explicações sobre a dinâmica, a temática a ser usada, as estratégias utilizadas, os assuntos tratados, materiais didáticos confeccionados, entre outros. Optou-se por criar um folder de linguagem simples e ilustrado com figuras divertidas (smurfs) e explicativas (acessos venosos), com o intuito de facilitar a compreensão das orientações e reforçar as informações transmitidas durante a ação.

O material utilizado como estratégia foi a confecção de dois tipos de bonecos de feltro: um grande $(49 \mathrm{~cm})$ para demonstração do acesso e de complicações; e o segundo foram 30 modelos menores $(15 X 10 \mathrm{~cm})$ para distribuição para as crianças, ambos apresentando o dispositivo venoso tipo jelco (sem agulha introdutora) para que a criança pudesse observar e manusear.

A terceira etapa foi a ação educativa, para abordar sobre o tema. Foram utilizadas metodologias ativas e o brinquedo terapêutico para condução da atividade. A dinâmica foi realizada em cinco momentos: no primeiro momento as acadêmicas se dirigiram a enfermaria para informar sobre a ação educativa. No segundo momento foi realizada a apresentação das acadêmicas e os objetivos da ação educativa com uma abordagem breve sobre o tema. No terceiro momento, houve a realização do quiz com os participantes que abordou: 1 O conceito de acesso venoso; 2- A finalidade do acesso venoso; 3 - Os locais que podem estar; 4- Os cuidados com o acesso venoso; 5- As complicações que podem ocorrer com o uso do acesso venoso. Após as perguntas/quiz, as acadêmicas mediaram a discussão para chegar à resposta correta. No quarto momento foi aberto um espaço para dúvidas e perguntas das crianças e seus acompanhantes sobre o tema. No quinto momento foi realizada a entrega e explicação do objetivo dos folders, e, também, distribuição dos brindes (smurfs de feltro pequeno).

A ação preconizava dois públicos alvos: pais/responsáveis e crianças, visto que no cenário pediátrico a idade da criança influencia na sua capacidade de autocuidado, assim, necessitando do cuidado de outra pessoa. Desse modo, participaram 24 responsáveis, dentre eles: pais, mães avós e tias, com idade variando de 18 a 48; e 24 crianças, 17 do sexo masculino e 7 do feminino - que podem foram agrupadas em duas categorias baseado na idade e no grau de dependência: de 01 mês a menores de 3 anos, no qual as 
orientações foram direcionadas para os acompanhantes; e de 3 a 14 anos, que receberam as orientações juntamente com os acompanhantes, interagindo durante a ação. Foi realizada nos quartos da própria enfermaria, compostas por três (3) leitos em cada quarto. Não há brinquedoteca na Instituição. A atividade durou em torno de duas (2) horas.

\section{DISCUSSÃO}

Quando se pensa no cuidado infantil, é essencial que a atenção seja integral, atendendo o lado biopsicossocial, além dos procedimentos, técnicas e medicações comumente realizados. Sua prática pode ocorrer na brinquedoteca, enfermaria, no leito, ou em outros locais, sempre com o intuito de tornar-se um espaço mais humanizado, afastando o estereótipo de medo que permeia o ambiente hospitalar, promovendo boa integração e passando confiança do enfermeiro (ou outro profissional de saúde) para a criança e a família (MARQUES DKA, et al., 2015).

Na pediatria, os pacientes apresentam maior grau de dependência de cuidados e a hospitalização causa impactos negativos na vida dessa população, o que demanda que a assistência prestada pela equipe de saúde à criança requer observação e cuidados durante seu planejamento e execução (FALKE ACS, et al., 2014)

No âmbito do cuidado à saúde da criança é de extrema importância a realização de atividades recreativas envolvendo o brincar, pois tal aspecto é necessário para o desenvolvimento infantil e deve ser mantido mesmo no ambiente hospitalar (OLIVEIRA CS, et al., 2015).

A partir da vivência da atividade, foi possível compreender que o uso do BT como ferramenta possui forte impacto para a criança no processo de hospitalização, sendo indispensável para proporcionar um cuidado humanizado para as crianças, visando o bem-estar físico, social e mental, além de alívio da ansiedade, descontração, relaxamento e conhecimento sobre si e seu estado atual. Florence Nightingale já reconhecia a importância do BT como estratégia na assistência de enfermagem ao paciente pediátrico. Ela utilizava histórias cantadas e jogos para ensinar sobre anatomia corporal e permitia períodos de recreação para esse público, uma vez que compreendia que por meio do brincar a criança consegue demonstrar suas emoções e sentimentos e se comunicar com outros indivíduos (BRASIL, 2005).

Apesar da existência de bases legais para sua prática, como a Resolução 41, de 13 de outubro de 1995 do Conselho Nacional dos Direitos da Criança e do Adolescente (CONANDA), que aborda os direitos da criança e do adolescente hospitalizados, prevê o direito desse público ser inserido em atividades lúdicas que auxiliem o seu desenvolvimento, mesmo em situações de internação e a Resolução 546/2017 que também regulamenta a utilização do BT pelos profissionais de enfermagem em sua prática, ainda não é uma realidade na maioria dos cenários pediátricos de saúde (BRASIL, 1995; COFEN, 2017).

Embora a relevância da temática, a existência de legislação que recomenda seu uso e a literatura apontar suas vantagens e benefícios, ainda se percebe uma reduzida adesão ao uso do BT como ferramenta estratégica para o cuidado, seja pelos profissionais e/ou pelos hospitais, que ainda priorizam a visão biologicista. Algumas dificuldades são apontadas pela literatura para seu emprego, tais como falta de tempo para atividade, falta de local apropriado e despreparo da equipe sobre o BT e seu uso. Para que de fato haja introdução na prática é necessário o reconhecimento do brincar, isso só ocorrerá através do embasamento teórica contemplados no curso de graduação, pós-graduação e nível técnico, a fim de desenvolver habilidades essenciais para o manejo objetivando desenvolver aproximação e relacionamento de confiança (MARQUES DKA, et al., 2015; CANÊZ JB, et al., 2019).

As Diretrizes Curriculares Nacionais destacam que a formação do enfermeiro deve ter a finalidade de formar profissionais críticos e reflexivos, que ainda no âmbito da graduação reconheçam a importância e apliquem na sua assistência a integralidade do cuidado às crianças, que considerem o brincar na assistência pediátrica, estabelecendo de uma comunicação ativa adequada a cada faixa etária (MAIA EBS, et al., 2019). É relevante que os profissionais de enfermagem incluam na sua prática os princípios humanísticos, para assim estabelecer a comunicação com a criança valorizem e promover uma assistência adequada (BARROSO MCCS, et al., 2019). 
A brinquedoteca hospitalar impacta diretamente no processo de tratamento e internação da criança, por estarem em um estado de ocorrem diversas transformações biopsicossocial, o lúdico influencia beneficamente e contribui como um excelente motivador para retomar a autoestima e diversão da criança. A instalação de brinquedotecas tem a finalidade de que a criança usufrua de um ambiente acolhedor e seguro, desvinculado do leito hospitalar, que seja capaz de amenizar sua angústia diante da internação hospitalar por meio da socialização entre a família e as outras crianças (SILVA C, et al., 2020). Entretanto, o hospital em questão não possui esse espaço, o que acaba prejudicando o tratamento por deixar a criança mais vulnerável ao sentimento de tristeza, medo e tensão pelo ambiente em que está inserida.

A qualidade do cuidado ofertado é essencial para manter a segurança do paciente. Desse modo, para realizar a instalação do acesso venoso, o enfermeiro necessita de diversos conhecimentos, tais como anatomia do sistema venoso, saber identificar a resposta fisiológica do sistema vascular, administração e a ação e efeito das drogas. Quando se trata da punção venosa em crianças, a segurança se redobra, é necessário considerar diversos fatores antes de determinar o local ideal: idade, tamanho, condição das veias, motivo da terapia, condições gerais, mobilidade e nível de atividade, habilidade motora fina e grossa da criança, percepção da imagem corporal, medo da mutilação e habilidade cognitiva. Um ambiente tranquilo e acolhedor facilita a aceitação da criança à realização do acesso venoso, ademais orientar a criança e o acompanhante sempre auxilia na execução do procedimento (ARROYO e LEITE, 2019).

Os familiares e acompanhantes são fundamentais no cuidado às crianças hospitalizadas, visto que elas ainda não compreendem os acontecimentos da internação. $O$ incentivo da participação efetiva do familiar no cuidado é de suma importância, tanto para fornecer carinho e apoio, como para ser parceiros da equipe no cuidado e criar a ponte entre o profissional e a criança. Esse elo favorece o tratamento, o processo de enfrentamento da situação e permite uma tranquila e segura experiência na internação hospitalar (PERES MA, et al., 2018). Ele ocorre através da transmissão de orientações educativas, cuidados direcionados à criança hospitalizada e familiares, além das ações técnicas destes profissionais de saúde, com foco no cuidado integral que são inerentes à vivência da criança e família no processo de hospitalização (AZEVÊDO AVS, et al., 2017).

Diante o exposto, foi notório os benefícios do emprego do BT como recurso para educar, tanto os responsáveis como às crianças hospitalizadas. Foi possível observar que a utilização do instrumento favoreceu a compreensão quanto importância do procedimento, e também, contribuiu para torná-los mais esclarecidos dos cuidados necessários com o acesso venoso periférico, aliado a isso, a estratégia favorece uma experiência de internação menos traumática. O BT é um direito da criança que está hospitalizada, portanto, é preciso capacitar e instrumentalizar a equipe de enfermagem e demais profissionais pediátricos para utilização da ferramenta de maneira adequada, visando usufruir das potencialidades da prática e reconhecimento das vantagens da implantação do brincar como forma de cuidar.

\section{REFERÊNCIAS}

1. ARROYO JCL, LEITE, SA. Acesso Venoso Possíveis em Pediatria. Jornada de Iniciação Científica do UNIFACIG. n. 5, 2019.

2. AZEVÊDO AVS, et al. Interação equipe de enfermagem, família, e criança hospitalizada: revisão integrativa. Ciênc. Saúde Coletiva, Rio de Janeiro, 2017; 22(11): 3653-3666.

3. BARROSO MCCS, et al. O brinquedo terapêutico na graduação de enfermagem: da teoria à prática. Rev Online de Pesquisa Cuidado é Fundamental, 2019; 11(4): 1043-1047.

4. BRASIL, Congresso Nacional. Lei Federal no 11.104 de 21 de março de 2005. Dispõe sobre a obrigatoriedade de instalação de brinquedotecas nas unidades de saúde que ofereçam atendimento pediátrico em regime de internação. Diário Oficial da República Federativa do Brasil, Brasília, DF, Seção 1, p 1.

5. BRASIL. Conselho Nacional dos Direitos da Criança e do Adolescente. Resolução n 41, de 13 de outubro de 1995. Aprova em sua íntegra o texto da Sociedade Brasileira de Pediatria, relativo aos Direitos da Criança e do Adolescente Hospitalizados. Brasília. 13 out. 1995

6. CALEFFI CCF, et al. Contribuição do brinquedo terapêutico estruturado em um modelo de cuidado de enfermagem para crianças hospitalizadas. Revista Gaúcha de Enfermagem. 2016; 37(2): e58131. 
7. CANÊZ JB, et al. O brinquedo terapêutico no cuidado à criança hospitalizada. Revista Enfermagem Atual In Derme, 2019; 88(26).

8. CONSELHO FEDERAL DE ENFERMAGEM. Resolução COFEN n. 0546/2017, de 9 de maio de 2017. Dispõe sobre a utilização da técnica do brinquedo terapêutico pela Equipe de Enfermagem na assistência à criança hospitalizada.

9. FALKE ACS, et al. Estratégias utilizadas pelos profissionais da enfermagem na abordagem a crianças hospitalizadas. Revista Contexto e Saúde, 2014; 18(34): 9-14.

10. JULIANI RML, SOUZA AS. O Uso do Brinquedo Terapêutico no Processo de Vacinação. Revista Pró-univerSUS. $2019 ; 10(1): 47-50$.

11. MAIA EBS, et al. Ensino do brinquedo terapêutico na graduação em enfermagem: ações e estratégias didáticas utilizadas por professores. Texto Contexto Enferm. 2019; 28: 1-17.

12. MARQUES DKA, et al. Benefícios da aplicação do brinquedo terapêutico: visão dos enfermeiros de um hospital infantil. Arq. Ciênc. Saúde. 2015; 22(3): 64-68.

13. OLIVEIRA CS, et al. Brinquedo Terapêutico na assistência à criança: percepção de enfermeiros das unidades pediátricas de um hospital universitário. Rev Soc Bras Enferm Ped. 2015; 15(1): 21-30.

14. PENNAFORTL VPS, et al. Brinquedo terapêutico instrucional no cuidado cultural da criança com diabetes tipo 1. Revista Brasileira Enfermagem. 2018; 71(3): 1415-23.

15. PERES MA, et al. Percepção de familiares e cuidadores quanto à segurança do paciente em unidades de internação pediátrica. Rev. Gaúcha Enferm. 2018; 39: e2017-0195.

16. SANTOS BRF, et al. Sistematização da assistência de enfermagem em isolamento respiratório pediátrico a fulgor da teoria das necessidades humanas básicas: relato de experiência. Revista Eletrônica Acervo Saúde, 2020; 50 : e2914.

17. SILVA C, et al. O enfermeiro e a criança: a prática do brincar e do brinquedo terapêutico durante a hospitalização. Semina: Ciências Biológicas e da Saúde. Londrina, 2020; 41(1): 95-106.

18. SILVA PR, et al. A importância do profissional da saúde na prevenção de infecção hospitalar causado por cateter venoso central. Mostra Interdisciplinar do Curso de Enfermagem. 2017.

19. ROCKEMBACH J, et al. Inserção do lúdico como facilitador da hospitalização na infância: percepção dos pais. J nurs health. 2017; 7(2): 117-26.

20. VEIGA MAB, et al. Enfermagem e o brinquedo terapêutico: vantagens do uso e dificuldades. Rev Eletrônica Atualiza Saúde. 2016; 3(3): 60-66. 